\title{
ÁREAS PROTEGIDAS DE CURITIBA (PR, BRASIL) COMO SUMIDOUROS DE $\mathrm{CO}_{2}$
}

\author{
Juliana Baladelli Ribeiroํㅗ Marília Borgo², Leila Teresinha Maranho³ \\ ${ }^{1}$ Bióloga, M.Sc., Secretaria Municipal de Meio Ambiente, SMMA, PMC, Curitiba, PR, Brasil - jubaladelli @ hotmail.com \\ ${ }^{2}$ Bióloga, Dr ${ }^{\mathrm{a}}$., The Nature Conservancy, TNC, Curitiba, PR, Brasil - maborgo@ yahoo.com \\ ${ }^{3}$ Bióloga, Dr ${ }^{\mathrm{a}}$., Programa de Pós-Graduação em Gestão Ambiental, UP, Curitiba, PR, Brasil - maranho@up.com.br
}

Recebido para publicação: 31/05/2012 - Aceito para publicação: 12/04/2013

\begin{abstract}
Resumo
A conservação de fragmentos florestais é extremamente necessária para garantir a manutenção da biodiversidade, além de assegurar o armazenamento de carbono contido na biomassa e nos solos. $\mathrm{O}$ município de Curitiba (PR, Brasil) possui 52 áreas protegidas municipais com remanescentes de Floresta Ombrófila Mista (FOM) em diferentes estágios de conservação, as quais contribuem para a manutenção da biodiversidade, melhoria da qualidade de vida dos moradores e também auxiliam na mitigação das mudanças climáticas, pois estocam grandes quantidades de carbono nos diversos componentes florestais. O presente estudo foi desenvolvido com o objetivo de avaliar a importância dos fragmentos florestais de Curitiba na manutenção dos estoques de carbono de forma associada à sua importância para a preservação da biodiversidade, o que pode servir de incentivo para o investimento público nessas áreas e de estímulo a outros municípios para a criação e manutenção de UCs. O estoque de carbono contido nos remanescentes florestais do município (1.159.259,60 t C) reforça a importância da conservação de remanescentes de vegetação nativa, ainda que em meio ao ambiente urbano.

Palavras-chave: Carbono; desmatamento evitado; Floresta com Araucária; áreas verdes urbanas.
\end{abstract}

\begin{abstract}
Protected Areas in Curitiba, PR, Brazil, as carbon sinks. Conservation of forest's fragments is extremely necessary to ensure maintenance of biodiversity and storage of carbon. There are currently 52 protected areas (PAs) in Curitiba municipality, located in Parana State - Brazil, with remnants of Araucaria Forest at different stages of conservation contributing to maintenance of biodiversity, improving quality of life of residents and also assisting in prevention of climate change as the aforementioned areas store large amounts of carbon in a range of forest components. In 2009, Curitiba conducted a research to estimate the carbon stock of forest fragments. The present research was carried out in order to evaluate the importance of these fragments in the maintenance of carbon stocks, associated to the preservation of biodiversity, which may be an incentive for public investment in these areas and encouragement to other municipalities for the creation and maintenance of PAs. Based on available information on the carbon contained in forest remnants of the city, it was identified that the total of those areas involves a stock of 1,159,259.60 t C, which reinforces the importance of maintaining native vegetation even in urban environment.
\end{abstract}

Keywords: Carbon; deforestation prevention; Araucaria Forest; urban protected areas.

\section{INTRODUÇÃO}

No Brasil, a principal fonte de emissão de gases de efeito estufa é o desmatamento (MACHADO, 2005; CARNEIRO, 2008; PINTO et al., 2009; MONZONI, 2009). Cardoso et al. (2009) afirmam que o desaparecimento de áreas naturais é uma das causas que mais contribuem para o aquecimento global. Além disso, a redução de áreas de floresta altera o ciclo hidrológico, com consequências que não são apenas locais, podendo afetar outros ecossistemas (MONZONI, 2009). Por exemplo, estima-se que entre 25 e $50 \%$ da chuva que chega ao Sudeste, região mais habitada e industrializada do Brasil, sejam provenientes do ciclo hidrológico da Bacia Amazônica, segundo Clement e Higuchi (2006). 
Evitar o desmatamento e recuperar áreas florestais são maneiras de mitigar as consequências das mudanças climáticas e também de garantir a manutenção de ecossistemas representativos, a conservação da biodiversidade, a qualidade das águas e a permeabilidade dos solos (CHANG, 2004; FERRETI, 2006; FERRETI; BRITEZ, 2006; CARDOSO et al., 2009). De acordo com Parker et al. (2009), o Brasil detém cerca de 80 bilhões de toneladas de carbono em florestas, quantidade equivalente à queima de combustíveis fósseis do mundo todo durante uma década.

Ecossistemas terrestres (solo e vegetação) podem ser considerados como sumidouros de carbono (SANQUETTA et al., 2003; MACHADO, 2005; SOMOGYI et al., 2006), devido ao potencial das florestas de "sequestrar" o $\mathrm{CO}_{2}$ durante seu desenvolvimento, reduzindo assim a concentração atmosférica desse gás de efeito estufa, retendo o carbono na biomassa e nos solos (SMITH et al., 2004; MACHADO, 2005; SILVEIRA et al., 2008; SOHGEN et al., 2008; CARDOSO et al., 2009). Dentro dos projetos do Mecanismo de Desenvolvimento Limpo (MDL), apenas os projetos florestais, de reflorestamento e florestamento, são capazes de capturar $\mathrm{CO}_{2}$ atmosférico. As demais modalidades de projetos conseguem apenas evitar ou reduzir as novas emissões (BRITEZ et al., 2006). Nesse caso, é possível aliar à mitigação das mudanças climáticas a necessidade de conservação dos ecossistemas, bem como dos serviços ambientais (FEARNSIDE, 2001; FEARNSIDE, 2008; CARDOSO et al., 2009; PARKER et al., 2009; MONZONI, 2009). A conservação da biodiversidade, da água e do solo, a inclusão social de comunidades locais com a criação de alternativas de renda e a recuperação de áreas degradadas são benefícios ambientais que devem ser associados ao valor das florestas nativas, que também acumulam carbono, além de outros serviços ambientais associados (MACHADO, 2005; BRITEZ et al., 2006; FERRETI; BRITEZ, 2006; CARDOSO et al., 2009).

A quantidade de carbono estocada em um fragmento florestal pode ser avaliada a partir do levantamento da biomassa, o que pode ser realizado por meio de diversas metodologias (BROWN et al., 1989; BROWN, 1997; AREVALO et al., 2002; KOEHLER et al., 2002; SANQUETTA, 2002; TIEPOLO et al., 2002; WATZLAWICK et al., 2002; WATZLAWICK et al., 2003; SMITH et al., 2004; CHAVE et al., 2005; BRITEZ et al., 2006; SOMOGYI et al., 2006; SILVEIRA et al., 2008; VIEIRA et al., 2008). Sanquetta (2002) e Sanquetta et al. (2003) afirmam ainda que existem relações lineares entre o peso verde da biomassa e o carbono presente nas diversas partes das árvores, e que as estimativas de carbono são obtidas com base na avaliação da biomassa por meio de inventários florestais, integrada ao mapeamento e medidas de área de determinada tipologia florestal.

A Floresta Ombrófila Mista (FOM), denominação atribuída pelo Instituto Brasileiro de Geografia e Estatística (IBGE, 1992), ou Floresta com Araucária (RODERJAN et al., 2002), é um dos ecossistemas associados ao Bioma Mata Atlântica (BRASIL, 2006). Cerca de 37\% da área do Paraná eram cobertos pela Floresta com Araucária (MAACK, 1981). A mudança de uso do solo e o crescente aumento da população ocuparam grande parte da sua área original, restando poucos trechos representativos dessa formação florestal, em diferentes estágios de regeneração, restando aproximadamente $0,8 \%$ de remanescentes em bom estado de conservação (CASTELLA et al., 2004).

O município de Curitiba possui remanescentes significativos de Floresta Ombrófila Mista, alguns abrigados em áreas protegidas públicas e outros em áreas particulares. A manutenção de áreas para o armazenamento de carbono é um serviço ambiental reconhecido em escala global, que pode ter um valor econômico considerável para países em desenvolvimento (RAMIREZ et al., 1999). A conservação dos fragmentos florestais, assim como a criação de corredores de ligação entre os fragmentos existentes, permite a manutenção da biodiversidade e garante o fluxo gênico entre as espécies, além de possibilitar a manutenção dos recursos hídricos, ao utilizar áreas de preservação permanente como corredores ecológicos (DURIGAN et al., 2006). O investimento na preservação de fragmentos florestais reflete ainda na qualidade de vida dos moradores, auxilia no balanço térmico, na regulação hídrica, previne erosão e deterioração do solo e preserva a biodiversidade (RAMIREZ et al., 1999; CHANG, 2004; FERRETI, 2006; FERRETI; BRITEZ 2006; SILVEIRA et al., 2008; CARDOSO et al., 2009).

A cidade investe na conservação de áreas verdes de domínio público e mantém um Sistema Municipal de Unidades de Conservação de Curitiba (SMUC, 2000), sob o qual estão os 21 Parques Naturais Municipais, 16 Bosques, um Jardim Botânico, duas Áreas de Proteção Ambiental e uma Estação Ecológica (SMMA, 2013). Até dezembro de 2012, havia 11 Reservas Particulares do Patrimônio Natural Municipais (RPPNMs) implantadas, de acordo com dados da SMMA. As áreas verdes a que este trabalho se refere foram definidas pelo artigo 20 da Lei Municipal 8353, de 22 de dezembro de 1993, como "os bosques de mata nativa representativos da flora do Município de Curitiba" (CURITIBA, 2000, on-line). 
Oficialmente, Curitiba apresenta $51,5 \mathrm{~m}^{2}$ de fragmentos florestais por habitante, uma das melhores médias do país, de acordo com Miguez (2001), que em seu trabalho utilizou a área total de 43.288,70 ha, sendo considerados 7.786,60 ha de área verde georreferenciada, o que representava aproximadamente $18 \%$ de área verde. Os imóveis com cobertura vegetal significativa são cadastrados pela SMMA e pertencem ao Setor de Áreas Verdes (SEAV), regidos pelo Decreto Municipal no 194 , de 2000 (CURITIBA, 2000), que prevê uma taxa de utilização específica para esses lotes, de acordo com a área, cobertura e tipologia florestal de cada um.

Durigan et al. (2006) estabeleceram indicadores a serem considerados na criação de áreas protegidas, os quais incluem tamanho, representatividade fitogeográfica, conectividade, proteção de mananciais, diversidade de fitofisionomias, riqueza de espécies lenhosas, número de espécies raras e endêmicas e quanto ao entorno (uso do solo no entorno e efeito de borda). O município de Curitiba levou em consideração, além desses fatores, a necessidade de proteção de mananciais, recursos hídricos, patrimônio histórico, belezas naturais e prevenção quanto às enchentes, implantando Parques Municipais em áreas consideradas de risco, criando lagos que funcionam como bacias de contenção, evitando alagamentos em pontos críticos da cidade (GILIOLI, 2006). Novos aspectos a serem considerados para a seleção de locais para a criação de áreas protegidas são as previsões de consequências das mudanças climáticas (SOHNGEN et al., 2008). De acordo com a International Union for Conservation of Nature (IUCN, 2001) e Araújo et al. (2004), as mudanças climáticas podem provocar migrações, podendo ocorrer o extremo de extinções localizadas em massa. Se houver mais áreas preservadas disponíveis, especialmente em zonas de transição entre ecossistemas, a possibilidade de adaptação e sobrevivência da maioria das espécies aumenta (ARAÚJO et al., 2004). Dessa maneira, além do estoque de carbono como meio para mitigação das mudanças climáticas, a manutenção de áreas com florestas nativas proveem uma série de outros serviços ambientais.

O principal objetivo do presente estudo é verificar a quantidade de carbono estocada nos fragmentos florestais em Curitiba e demonstrar o potencial das áreas protegidas, bem como sumidouros de $\mathrm{CO}_{2}$, aliado à conservação da biodiversidade.

\section{MATERIAIS E MÉTODOS}

Foram analisados os resultados de quantificação de carbono do "Programa de avaliação e quantificação do potencial de absorção de carbono por florestas nativas em Curitiba", realizado por meio de parceria entre a Prefeitura Municipal de Curitiba e a Sociedade de Pesquisa em Vida Selvagem e Educação Ambiental (SPVS, 2009). As informações obtidas foram confrontadas com dados do Centro de Geoprocessamento da Secretaria Municipal de Meio Ambiente (SMMA) da cidade referentes às áreas com fragmentos florestais contínuos superiores a $2.000 \mathrm{~m}^{2}$, conforme a tipologia florestal utilizada por esse Centro de Geoprocessamento para cadastro dos fragmentos florestais definida oficialmente, com base em Miguez (2001), o qual classificou a vegetação em: 1) Mata nativa com araucária; 2) Mata nativa sem araucária; 3) Mata secundária em regeneração (capoeira); 4) Bracatingal; 5) Mata ciliar; 6) Reflorestamento.

Em cada uma das áreas selecionadas, foram instaladas parcelas circulares de $10 \mathrm{~m}$ de raio, em número variável, preferencialmente afastadas das áreas de visitação pública e sobre terreno com relevo uniforme. Os dados referentes ao PAP, à distância do indivíduo até o centro da parcela e à numeração da plaqueta foram registrados em fichas de campo. Para as palmeiras e xaxins, foi utilizado o mesmo critério de inclusão, porém anotadas apenas as informações referentes à altura dos indivíduos, pois a biomassa desses grupos de plantas tem relação alométrica com esse parâmetro e não com o diâmetro. A medição da altura das palmeiras foi feita considerando-se o comprimento total do estipe, popularmente chamado de tronco. A medida de distância da árvore até o centro da parcela foi verificada com equipamento especializado, denominado "DME" (Distance Measuring Equipment).

As estimativas de carbono realizadas por SPVS (2009) pautaram-se em amostragem indireta em áreas protegidas municipais, que consiste em levantamento fitossociológico de parcelas dentro dos fragmentos florestais remanescentes (MACDICKEN, 1997). Os resultados obtidos fornecem subsídios para os cálculos de quantidade de carbono na biomassa, com o uso de equações de regressão conforme Tiepolo et al. (2002). A equação utilizada pela equipe responsável pela medição do carbono (SPVS, 2009) foi a descrita na tabela 1. A utilização de amostragem direta, que prevê o corte de indivíduos da vegetação nativa, causaria grande impacto tanto nos remanescentes, que via de regra são pouco extensos, 
quanto na população em geral, pois qualquer ação de intervenção em trechos com vegetação na cidade é vista como prejudicial ao senso comum de conservação.

Tabela 1. Equações utilizadas por SPVS (2009) na estimativa da biomassa vegetal aérea.

Table 1. Equations used by SPVS (2009) to estimate aerial vegetal biomass.

\begin{tabular}{lccc}
\hline Categoria & Equação & $\mathbf{R}^{\mathbf{2}}$ & Referências \\
\hline Árvores & $\mathrm{Y}=21,297-6,953(\mathrm{DAP})+0,74\left(\mathrm{DAP}^{2}\right)$ & 0,91 & Brown, 1997 \\
Palmeiras & $\mathrm{Y}=0,3999+7.907 *$ altura & 0,75 & Tiepolo et al., 2002 \\
Xaxins & $\mathrm{Y}=(1563,547 * \mathrm{EXP}(0,310478 *$ altura $)) / 1000$ & 0,93 & Tiepolo et al., 2002 \\
\hline
\end{tabular}

Foi realizada a comparação do levantamento feito pela SPVS para a Prefeitura Municipal de Curitiba (SPVS, 2009) com trabalhos semelhantes em outras localidades, porém no mesmo bioma, como os estudos de Tiepolo et al. (2002), Watzlawick et al. (2002), Watzlawick et al. (2003), Britez et al. (2006), Caldeira et al. (2007), Vieira et al. (2008) e Cardoso et al. (2009).

\section{RESULTADOS E DISCUSSÃO}

Confrontando-se os dados da SPVS (2009) com as informações sobre cobertura vegetacional disponibilizados por Miguez (2001), foi estimado um total de 1.159.260 toneladas de carbono contidas nos remanescentes florestais da cidade (Tabela 2). Quase 80\% desse valor referem-se a áreas de floresta nativa com araucária em estágio médio e avançado, tipologia com a maior quantidade de carbono estocado por unidade de área (SPVS, 2009). Coincidentemente, essa fase sucessional representa a vegetação mais antiga, corroborando a perspectiva de que as maiores quantidades de carbono encontramse justamente nessas condições, onde o processo de sucessão permitiu o acúmulo de matéria orgânica (e, dessa forma, de carbono) ao longo do tempo (WATZLAWICK et al. 2002; FERRETI; BRITEZ, 2006).

Tabela 2. Distribuição dos estoques de carbono entre as diferentes formações vegetacionais encontradas em Curitiba, Paraná, Brasil.

Table 2. Distribution of carbon storage in different forest stagesin Curitiba Municipality, Paraná, Brazil.

\begin{tabular}{lccc}
\hline Classe vegetacional & Área (ha)* & $\begin{array}{c}\text { Carbono } \\
\left(\mathbf{t ~ C . h a ~}^{-\mathbf{1}}\right)^{* *}\end{array}$ & $\begin{array}{c}\text { Estoque carbono } \\
(\mathbf{t ~ C )}\end{array}$ \\
\hline Floresta nativa com araucária & $5.462,1$ & 168,25 & $918.998,32$ \\
Floresta nativa sem araucária & $1.092,1$ & 168,25 & $183.745,82$ \\
Floresta secundária & 269,1 & $97,81 /$ & $26.320,67$ \\
Bracatingal & 229,5 & 56,26 & $12.911,67$ \\
Reflorestamento & 511,8 & - & não incluído \\
Floresta ciliar & 213,9 & 80,80 & $17.283,12$ \\
\hline Total & & & $1.159 .259,60$ \\
\hline
\end{tabular}

Fonte: * Miguez (2001); ** SPVS (2009).

Essa constatação reforça a relevância da manutenção de florestas nativas em meio urbano, pois, ainda que submetidas às mais variadas intervenções, em diferentes condições de fragmentação e sujeitas às alterações impelidas pela presença da população humana, preservam quantidades bastante significativas de carbono.

As quantidades de carbono encontradas nos remanescentes nativos de Curitiba não fogem às estimativas gerais estabelecidas para a Floresta com Araucária, como pode ser constatado nos estudos conduzidos por Britez et al. (2006) e Cardoso et al. (2009). Nesses trabalhos, os estoques de carbono variaram entre 29 t C.ha ${ }^{-1}$ e 176 t C.ha ${ }^{-1}$ para áreas alteradas e intensamente exploradas em diferentes estágios de regeneração. Os valores de carbono encontrados nas florestas urbanas assemelharam-se bastante aos encontrados por Watzlawick et al. (2002), que realizaram estudo em diferentes estágios sucessionais de FOM no município de General Carneiro, PR, e encontraram 165,02 t C.ha ${ }^{-1}$ para o estágio avançado.

Cardoso et al. (2009) utilizaram as equações propostas por Brown (1997) para estimar a quantidade de $\mathrm{C}$ presente em áreas de FOM e chegaram ao valor de aproximadamente $120 \mathrm{t} \mathrm{C}^{-\mathrm{ha}^{-1}}$ para 
florestas em estágio avançado. Já Watzlawick et al. (2002) encontraram valores diferenciados para a biomassa nos diferentes estágios sucessionais de Floresta Ombrófila Mista (FOM) em General Carneiro, sendo 168,84 t.ha $^{-1}$ para estágio médio e 397,79 t.ha $^{-1}$ para estágio avançado. Essa metodologia considera árvores com DAP $>10 \mathrm{~cm}$, na mesma tipologia florestal. O estágio avançado continha 5,7 vezes mais $\mathrm{C}$ que o inicial, e 2,3 vezes mais carbono que o estágio intermediário.

Entre as medidas de mitigação às mudanças climáticas que estão sendo adotadas em Curitiba, podem ser destacadas justamente as ações de manutenção e recuperação dessas áreas naturais, tendo em vista que elas representam importantes fontes de absorção e acúmulo de carbono atmosférico. A criação de parques municipais nas Áreas de Preservação Permanente (APPs) dos maiores rios da cidade é um exemplo disso, onde o estabelecimento de UCs e a realocação de famílias que vivem nas Áreas de Preservação Permanente evita a sua ocupação irregular, diminuindo a possibilidade de poluição, assoreamento e lançamentos irregulares de efluentes, entre outros impactos ambientais negativos. Tal ferramenta acaba por garantir a proteção do entorno das nascentes, mantendo a permeabilidade das APPs e possibilitando, assim, a recarga do lençol freático, além de garantir melhor qualidade de vida para os moradores, fatores considerados importantes para a sustentação das funções ecológicas das florestas ribeirinhas por Socher et al. (2007). Além das ações de manutenção dos trechos já existentes, que asseguram a não liberação do carbono fixado pela vegetação para a atmosfera, as ações de recuperação das APPs, inseridas ou não em Unidades de Conservação, irão propiciar a captura de carbono pela vegetação ao longo do tempo.

Do total de trechos com vegetação nativa georreferenciados em Curitiba (Tabela 3), 1.944,18 ha são considerados áreas oficiais em áreas protegidas e, portanto, têm assegurada a sua preservação. Além da manutenção das áreas protegidas, há que se considerar a possibilidade de aumentar ou interligar as áreas já existentes, ou ainda priorizar áreas de transição entre ecossistemas com características ambientais diferentes, visto que, como afirmam Araújo et al. (2004) e Campos (2006), a distribuição das espécies não é homogênea, e áreas de transição garantem maior mobilidade à fauna em ambientes sob constantes modificações.

Tabela 3. Estimativa de carbono nos fragmentos florestais em Curitiba.

Table 3. Estimate of carbon in forest areas in Curitiba.

\begin{tabular}{lcc}
\hline Classificação & Área (ha) & Estimativa de carbono (t C) \\
\hline Área verde georreferenciada & 7.778 & 1.159 .259 \\
Unidades de Conservação & 1.944 & 327.109 \\
Imóveis particulares & 5.834 & 981.640 \\
Imóveis passíveis de criação de RPPNM & 1.899 & 319.533 \\
\hline
\end{tabular}

Outro aspecto importante envolve a substituição das árvores exóticas invasoras da arborização pública por espécies nativas do ecossistema local como parte do Programa Biocidade, implantado pela Prefeitura Municipal de Curitiba, medida essa que também pode ser incluída entre as medidas que visam combater as mudanças climáticas, visto que, além de capturar carbono atmosférico durante seu desenvolvimento, as árvores auxiliam na manutenção da qualidade do ambiente urbano, com redução das ilhas de calor e incremento de beleza cênica, oferecem abrigo, alimentação e nidificação para a fauna e ainda armazenam carbono na fitomassa e no solo.

O restante, que representa $75 \%$ dos remanescentes encontrados, encontra-se em imóveis particulares, que têm seu uso restrito pela legislação vigente. Seguindo as prerrogativas legais, que permitem uma taxa de utilização de até $30 \%$ em cada um desses imóveis, o uso dessas áreas resultaria na emissão para a atmosfera de 294.492,34 t C. Além da perda associada ao uso permitido da floresta, perdese também o potencial contínuo de absorção de carbono pela vegetação através do processo fotossintético. Soma-se a isso a depauperação da qualidade ambiental deflagrada pela intensificação do efeito de borda nesses locais, em consequência da maior fragmentação, que afeta negativamente a produtividade do sistema biológico, interferindo, dessa forma, nos valores de acúmulo de carbono (PRIMACK; RODRIGUES, 2001; MEFFE; CARROLL, 1994).

Tendo em vista a representatividade desses remanescentes contidos em imóveis particulares, é de extrema importância o desenvolvimento de mecanismos que priorizem a conservação dessas áreas. Um deles é a possibilidade de criação de Reservas Particulares do Patrimônio Natural Municipais (RPPNMs), 
categoria de Unidade de Conservação pioneira do município de Curitiba que permite ao proprietário de área preservada manter sua área e ainda obter a transferência do potencial construtivo para outros imóveis, preservando a vegetação, de acordo com a legislação vigente (RIBEIRO; MORATO, 2009). Existem mais de 1.000 imóveis em Curitiba (1.899 ha) onde a vegetação apresenta características que permitem a aplicação desse mecanismo, e já existem quatro RPPNMs criadas, além de 23 em processo de análise na SMMA (comunicação pessoal, Centro de Geoprocessamento SMMA). Além de garantir corredores de biodiversidade no meio urbano, resguardar esses imóveis ainda torna possível evitar a emissão de 319.534 t C. Em longo prazo, o valor econômico do serviço ambiental de armazenamento de carbono, que pode ser associado ao manejo sustentável e uso controlado, pode ser maior que o valor financeiro da exploração da área, conforme Ramirez et al. (1999) confirmaram em seu estudo.

O aprimoramento dos mecanismos já existentes, bem como a criação de novos incentivos à conservação de áreas naturais, é essencial para que esse cenário traga uma maior contribuição à agenda da Convenção do Clima. A criação de novas ferramentas legais que estabeleçam áreas prioritárias para a conservação entre esses imóveis é um dos caminhos a ser trilhado, e pode ser pautado em critérios específicos, como os sugeridos por Durigan et al. (2006) e Cardoso et al. (2009), para que, além da capacidade de absorção e armazenamento de carbono, sejam também considerados o tamanho, a conectividade entre fragmentos, a existência de recursos hídricos, a proximidade a UCs, a constatação de elementos de fauna ou flora ameaçados de extinção, a integridade da vegetação e possibilidades de regeneração, sua localização como ambiente de transição, observando ainda dificuldades financeiras do proprietário na manutenção de área com vocação para a conservação.

Em estudo realizado por Bertolo et al. (2005), foram determinados valores diferenciados de cobertura das áreas verdes de Curitiba com o uso de imagens Landsat TM de 1986, 1999 e 2002. Segundo as autoras, que consideraram como agrupamentos arbóreos as matas remanescentes, matas ciliares e os reflorestamentos, sem incluir áreas abertas de pastagens, culturas ou qualquer outro tipo de atividade agrícola, o aumento da vegetação que foi verificado ao longo dos anos ocorreu principalmente devido aos avanços das políticas públicas urbanas. Os mapeamentos realizados mostraram que entre 1986 e 2002 houve um acréscimo de $9 \%$ na cobertura vegetal do município. Porém sugere-se que novos estudos devem ser contratados pelo poder público para revisão dos números oficiais de cobertura vegetal, utilizando-se de imagens de satélite e tecnologias mais precisas.

\section{CONCLUSÕES}

- Os fragmentos de florestas urbanas de Curitiba, apesar de suas áreas relativamente pequenas, interferência antrópica e uso público, apresentam características semelhantes às florestas naturais em termos de estoque de carbono, motivo pelo qual são importantes para o armazenamento de carbono. E mesmo que constituam uma pequena fração dentro das possíveis medidas de mitigação aos efeitos das mudanças climáticas em grandes cidades, sua existência e manutenção deve ser amplamente valorizadas, uma vez apresentam grande importância para a conservação da biodiversidade, na composição de mosaicos ou como trampolins ecológicos, o que facilita o fluxo gênico de espécies vegetais e animais.

- Além da capacidade de armazenar carbono na biomassa, essas áreas agregam valores relacionados à manutenção do patrimônio biológico, que podem amplificados e difundidos pelo desenvolvimento de estudos, pesquisas científicas e atividades de Educação Ambiental, além de proverem qualidade ambiental aos moradores dos centros urbanos no que se refere à qualidade, quantidade e distribuição de espaços livres que permitam um contato saudável com a natureza.

- A viabilização de mecanismos efetivos de manutenção desses locais é fundamental dentro de uma condição de políticas públicas que envolvam responsabilidade com os cidadãos e com o meio ambiente. Evitar o desmatamento é uma das formas mais eficientes de mitigar as consequências das mudanças climáticas em países como o Brasil, além de garantir a manutenção de ecossistemas representativos e biomas extremamente ameaçados, como é o caso da Mata Atlântica.

- As informações aqui apresentadas podem incentivar o investimento público nessas áreas e servem de estímulo a outros municípios para a criação e manutenção de UCs, que, além de atenderem aos motivos que justificam a sua criação, contribuem com a manutenção do estoque de carbono e outros serviços ambientais. 


\section{REFERÊNCIAS}

ARAÚJO, M. B.; CABEZA, M.; THUILLER, W.; HANNAH, L.; WILLIAMS, P. H. Would climate change drive species out of reserves? An assessment of existing reserve-selection methods. Global Change Biology, n. 10, p. 1618 - 1626, 2004.

AREVALO, L. A.; ALEGRE, J. C.; VILCAHUAMAN, L. J. M. Metodologia para estimar o estoque de carbono em diferentes sistemas de uso da terra. Colombo, EMBRAPA Florestas, 2002.

BERTOLO, S. L.; ROCHA, J. V.; YOUNG, A. F. Evolução temporal do índice de vegetação da área urbana de Curitiba, PR. Goiânia, Brasil, Simpósio Brasileiro de Sensoriamento Remoto, INPE 2005.

BRASIL. Lei Federal $\mathrm{n}^{\circ} 11.428$ de 22 de dezembro de 2006 . Disponível em <http://www.planalto.gov.br/ccivil_03/_Ato2004-2006/2006/Lei/L11428.htm>. Acesso em: 24/04/2012.

BRITEZ, R. M.; BORGO, M.; TIEPOLO, G.; FERRETI, A.; CALMON, M.; HIGA, R. Estoque e incremento de carbono em florestas e povoamentos de espécies arbóreas com ênfase na Floresta Atlântica do sul do Brasil. Colombo: Embrapa Florestas, 2006.

BROWN, S.; GILLESPIE, A. J. R.; LUGO, A. E. Biomass estimation methods for Tropical Forests with applications to forest inventory data. Forest Science, v. 35, n. 4, p. 881 - 902, 1989.

BROWN, S. Estimating biomass and biomass change of tropical forests: a primer. Roma, Itália: FAO Forestry Paper 134. 1997. Disponível em <http://www.fao.org/docrep/w4095e/w4095e00.htm>. Acesso em: 24/04/2012.

CALDEIRA, M. V. W.; MARQUES, R.; SOARES, R. V.; BALBINOT, R. Quantificação de serapilheira e de nutrientes - Floresta Ombrófila Mista Montana - Paraná. Revista Acadêmica, v. 5, n. 2, 2007.

CAMPOS, J. B. A fragmentação de ecossistemas, efeitos decorrentes e corredores de biodiversidade. In: CAMPOS, J. B.; TOSSULINO, M. G. P.; MÜLLER, C. R. C. (Org.). Unidades de Conservação: ações para a valorização da biodiversidade. Curitiba: IAP, v. 1, p. 165 - 173, 2006.

CARDOSO, D. N.; KARAM, G. Z.; CONEGLIAN, S. J. G.; HOFFMANN, P. M.; SANTOS, J. J. S.; KRUGER, F. A. Programa Desmatamento Evitado (SPVS): um mecanismo complementar ao SNUC para a conservação em áreas privadas. In: VI Congresso Brasileiro de Unidades de Conservação, Anais em CD, v.1 Trabalhos Técnicos. Curitiba: Fundação O Boticário de Proteção à Natureza/Rede Nacional Pró-Unidades de Conservação, 2009.

CARNEIRO, P. E. A. Modelo de mudanças climáticas com gastos públicos. Rio de Janeiro: Contexto Internacional, v. 30, n. 1, p. 1 - 20. 2008.

CASTELLA, P. R.; BRITEZ, R. M.; MIKICH, S. B. Áreas prioritárias de Floresta com Araucária para conservação no Estado do Paraná. In: IV Congresso Brasileiro de Unidades de Conservação, Anais, v. 1 Trabalhos Técnicos. p. 134 - 143. Curitiba: Fundação O Boticário de Proteção à Natureza/Rede Nacional Pró-Unidades de Conservação, 2004.

CHANG, M. Y. Sequestro florestal de carbono no Brasil - dimensões políticas, socioeconômicas e ecológicas. In: SANQUETTA, C. R.; BALBINOT. R.; ZILIOTTO, M. A. B. Fixação de Carbono: Atualidades, Projetos e Pesquisas. Curitiba: Laboratório de Inventário Florestal - UFPR/Instituto Ecoplan, p. 15 - 37, 2004.

CHAVE, J.; ANDALO, C.; BROWN, S.; CAIRNS, M. A. C.; CHAMBERS, J. Q.; EAMUS, D.; FÖLSTER, H.; FROMARD, F.; HIGUCHI, N.; KIRA, T.; LESCURE, J.-P.; NELSON, B. W.; OGAWA, H.; PUIG, H.; RIERA, B.; YAMAKURA, T. Tree allometry and improved estimation of carbon stocks and balance in tropical forests. Oecologia, v. 145, p. 87 - 99, 2005.

CLEMENT, C. R.; HIGUCHI, N. A Floresta amazônica e o futuro do Brasil. Ciência e Cultura, v. 58, n. 3, p. $44-49,2006$. 
CONAMA. Conselho Nacional de Meio Ambiente. Resolução no 02 de 18 de março de 1994. Disponível em <http://www.mma.gov.br/port/conama/res/res94/res0294.html>. Acesso em: 24/04/2012.

CURITIBA. Lei Municipal no 9806 de 03 de janeiro de 2000 e Decreto Municipal no 194 de 2000. Disponível em <http://www.curitiba.pr.gov.br/multimidia/00086314.pdf>. Acesso em: 24/04/2012.

DURIGAN, G.; SIQUEIRA, M. F.; FRANCO, G. A. D. C.; RATTER, J. A. Seleção de fragmentos prioritários para a criação de Unidades de Conservação do Cerrado no Estado de São Paulo. Revista do Instituto Florestal, São Paulo. v. 18, n. único, p. 23 - 37, 2006.

FEARNSIDE, P. M. As florestas no acordo do clima. Ciência Hoje, v. 29, n. 171, p. 60 - 62, 2001.

FEARNSIDE, P. M. Quantificação do serviço ambiental do carbono nas Florestas Amazônicas Brasileiras. Oecologia Brasileira, v. 12, n. 4, p. 743 - 756, 2008.

FERRETI, A. R. O papel das Unidades de Conservação no combate às mudanças climáticas e as oportunidades geradas. In: IAP Unidades de Conservação - Ações para Valoração da Biodiversidade, p. 153 - 162, 2006.

FERRETI, A. R.; BRITEZ, R. M. Ecological Restoration, Carbon Sequestration and Biodiversity Conservation: the experience of the Society for Wildlife Research and Environmental Education (SPVS) in the Atlantic Rain Forest of Southern Brazil. Journal for Nature Conservation, p. 1 - 11, 2006.

GILIOLI, L. N. Parques e Bosques em Curitiba no Paraná. 2006. 29 f. Dissertação de Mestrado em Programação dos Ambientes Urbanos Sustentáveis. Pontifícia Universidade Católica do Paraná. PUC-PR, 2006.

INSTITUTO BRASILEIRO DE GEOGRAFIA E ESTATÍSTICA (IBGE). Departamento de Recursos Naturais e Estudos Ambientais. Manual Técnico da Vegetação Brasileira. Série Manuais Técnicos em Geociências, n. 1. Rio de Janeiro, 1992.

INTERNATIONAL UNION FOR CONSERVATION OF NATURE (IUCN). Implications of climate change for species conservation - Briefing Paper. IUCN Workshop, Gland, Switzerland. February, 2001.

IPCC. Intergovernmental Panel on Climate Change. Disponível em <http://www.ipcc.ch/>. Acesso em: 24/04/2012.

KOEHLER, H. S.; WATZLAWICK, L. F.; KIRCHNER, F. F. Fontes e níveis de erros nas estimativas do potencial de fixação do carbono. In: SANQUETTA, C. R.; WATZLAWICK, L. F.; BALBINO, T. R.; ZILIOTTO, M. A. B.; GOMES, F. S. As florestas e o carbono, Curitiba, Universidade Federal do Paraná, p. 119 - 140, 2002.

MAACK, R. Geografia Física do Estado do Paraná. 2. ed. Rio de Janeiro: José Olympio, 1981.

MACHADO, P. L. O. A. Carbono do solo e a mitigação da mudança climática global. São Paulo: Química Nova, v. 28, n. 2, p. 329 - 334, 2005.

MACDICKEN, K. G. A Guide to Monitoring Carbon Storage in Forestry and Agroforestry Projects. Forest Carbon Monitoring Program. Winrock International Institute for Agricultural Development, 1997. Disponível em: <http://www.forestcarbonportal.com/resource/guide-monitoringcarbon-storage-forestry-and-agroforestry-projects>. Acesso em: 26/02/2013.

MEFFE, G. K.; CARROLL, C. R. Principles of conservation biology. Massachussets: Sinauer, 1994. $600 \mathrm{p}$.

MIGUEZ, L. A. Mapeamento e monitoramento dos maciços vegetais do município de Curitiba, PR. 2001. 36f. Monografia (Especialização em Qualidade de Vida Urbana) - Faculdade de Administração e Economia do Paraná (FAEP), Instituto de Engenharia do Paraná (IEP), 2001.

MONZONI, M. Diretrizes para formulação de Políticas Públicas em Mudanças Climáticas no Brasil. Observatório do Clima e Centro de Estudos em Sustentabilidade da EAESP/FGV, 2009. 
PARKER, C.; MITCHELL, A.; TRIVEDI, M.; MARDAS, N. The little REDD+ Book. Global Canopy Foundation, 2009.

PINTO E. P. P.; MOUTINHO, P.; RODRIGUES, L.; FRANÇA, O. F. G.; MOREIRA, P. F.; DIETZSCH, L. Perguntas e respostas sobre o Aquecimento Global. Belém: Instituto de Pesquisa Ambiental da Amazônia - IPAM/ The Woods Hole Research Center, 2009. 63 p.

PRIMACK, R. B.; RODRIGUES, E. Biologia da Conservação. Londrina: E. Rodrigues, 2001. 327 p.

RAMÍREZ, O. A.; RODRIGUEZ, L.; FINEGAN, B.; GIMENEZ, M. Implicaciones economicas del secuestro del $\mathrm{CO}_{2}$ em bosques naturales. Turrialba: Revista Forestal Centroamericana, n. 27, p. 10 - 16, 1999.

RIBEIRO, J. B.; MORATO, S. A. A. Roteiro metodológico para elaboração do Plano de Manejo de Reservas Particulares do Patrimônio Natural Municipais em Curitiba-PR, Brasil. In: VI Congresso Brasileiro de Unidades de Conservação, Anais em CD, v. 1. Trabalhos Técnicos. Curitiba: Fundação O Boticário de Proteção à Natureza/Rede Nacional Pró-Unidades de Conservação, 2009.

RODERJAN, C. V.; GALVÃO, F.; KUNIYOSHI, Y. S.; HATSCHBACH, G. G. As Unidades Fitogeográficas do Estado do Paraná. Ciência e Ambiente, n. 24, 2002.

SANQUETTA, C. R. Métodos de determinação de biomassa florestal. In: SANQUETTA, C. R.; WATZLAWICK, L. F.; BALBINO, T. R.; ZILIOTTO, M. A. B.; GOMES, F. S. As florestas e o carbono. Curitiba, Universidade Federal do Paraná, p. 119 - 140, 2002.

SANQUETTA, C. R.; WATZLAWICK, L. F.; SCHUMACHER, M. V.; MELlO, A. A. Relações individuais de biomassa e conteúdo de carbono em plantações de Araucaria angustifolia e Pinus taeda no sul do estado do Paraná, Brasil. Revista Acadêmica: ciências agrárias e ambientais, v. 1, n. 3. p. 33 - 40, 2003.

SECRETARIA MUNICIPAL DO MEIO AMBIENTE (SMMA), Curitiba, PR. Disponível em: $\langle$ http://www.curitiba.pr.gov.br/conteudo/parques-e-bosques-smma-secretaria-municipal-do-meio-ambiente/267>. Acesso em: 25/02/2013.

SILVEIRA, P.; KOEHLER, H. S.; SANQUETTA, C. R.; ARCE, J. E. O estado da arte na estimativa de biomassa e carbono em formações florestais. Floresta, v. 38, n. 1, p. 185 - 206, 2008.

SISTEMA MUNICIPAL DE UNIDADES DE CONSERVAÇÃO DE CURITIBA PR (SMUC). Lei Municipal $\mathrm{n}^{\circ} 9804$ de 03 de janeiro de 2000. Disponível em <http://www.curitiba.pr.gov.br/ multimidia/00086311.pdf>. Acesso em: 24/04/2012.

SMITH, J. E.; HEATH, L. S.; WOODBURY, P. B. How to estimate forest carbon for large areas from inventory data. Journal of Forestry, v. 102, p. 25 - 31, 2004.

SOCHER, L. G.; RODERJAM, C. V.; GALVÃO, F. Biomassa aérea de uma Floresta Ombrófila Mista Aluvial no município de Araucária, PR. Floresta, v. 38, n. 2, p. 245 - 252, 2007.

SOHNGEN, B.; BEACH, R. H.; ANDRASKO, K. Avoided Deforestation as a Greenhouse Gas Mitigation Tool. Journal of Environmental Quality, v. 37, p. 1368 - 1375, 2008.

SOCIEDADE DE PESQUISA EM VIDA SELVAGEM E EDUCAÇÃO AMBIENTAL (SPVS). Programa de avaliação e quantificação do potencial de absorção de carbono por florestas nativas em Curitiba. Relatório Final de Atividades. SPVS/SMMA. 2009.

SOMOGYI, Z.; CIECIALA, E.; MAKIPAA, R.; MUUKKONEN, P.; LEHTONEN, A.; WEISS, P. Indirect methods of large-scale forest biomass estimation. Europe Journal Forest Research, v. 126, n. 2, p. $197-207,2006$.

TIEPOLO, G.; CALMON, M.; FERETI, A. R. Measuring and monitoring carbon stocks at the Guaraqueçaba Climate Action Project, Paraná, Brazil. In: International Symposium on Forest Carbon Sequestration and Monitoring. Taiwan Forestry Research Institute, n. 153. p. 98 - 115, 2002. 
VIEIRA, S. A.; ALVES, L. A.; AIDAR, M.; ARAÚJO, L. S.; BAKER, T.; BATISTA, J. L. F.; CAMPOS, M. C.; CAMARGO, P. B.; CHAVES, J.; DELITTI, W. B. C.; HIGUCHI, N.; HONORIO, E.; JOLY, C. A.; KELLER, M.; MARTINELLI, L. A.; MATTOS, E. A.; METZKER, T.; PHILLIPS, O.; SANTOS, F. A. M.; SHIMABUKURO, M. T.; SILVEIRA, M.; TRUMBORE, S. E. Estimation of biomass and carbon stock: the case of the Atlantic Forest. Fapesp: Biota Neotropica, v. 8, n. 2, p. 21 - 29 , 2008.

VIEIRA, C. H. S. D.; BIONDI, D. Análise da dinâmica da cobertura vegetal de Curitiba, PR (de 1986 a 2004), utilizando imagens LANDSAT TM1. Revista Árvore, v. 32, n. 3, 2008.

WATZLAWICK, L. F.; KIRCHNER, F. F.; SANQUETTA, C. R.; SCHUMACHER, M. V. Fixação de carbono em Floresta Ombrófila Mista em diferentes estágios de regeneração. In: SANQUETTA, C. R.; WATZLAWICK, L. F.; BALBINO, T. R.; ZILIOTTO, M. A. B.; GOMES, F. S. As florestas e o carbono. Curitiba, Universidade Federal do Paraná, p. 119 - 140, 2002.

WATZLAWICK, L. F.; SANQUETTA, C. R; ARCE, J. E.; BALBINOT, R. Quantificação de biomassa total e carbono orgânico em povoamentos de Araucaria angustifolia (Bert.) O. Kuntze no sul do estado do Paraná, Brasil. Curitiba: Revista Acadêmica: ciências agrárias e ambientais, v. 1, n. 2, p. 63 - 68, 2003. 